\title{
Hyperseparoids: A Representation Theorem
}

\author{
Ricardo Strausz
}

Received: 15 January 2010 / Revised: 14 July 2010 / Accepted: 25 August 2010 /

Published online: 29 September 2010

(C) Springer Science+Business Media, LLC 2010

\begin{abstract}
In this note, hyperseparoids are introduced; hyperseparoids are to separoids as Tverberg's theorem is to Radon's theorem. Also, a geometric representation theorem for acyclic k-separoids is presented which generalises that for separoids exhibited in Bracho and Strausz (Period. Math. Hung. 53:115-120, 2006).
\end{abstract}

Keywords Separoids · Tverberg's theorem · Radon's theorem · Abstract convexity · Relational systems · Combinatorial geometry

A separoid is a (finite) set $S$ endowed with a symmetric relation $\dagger \subseteq\left(\begin{array}{c}2^{S} \\ 2\end{array}\right)$, defined in its family of subsets, which satisfies the following simple properties (cf. [1-7]): for $A, B \subseteq S$

$$
\begin{aligned}
& \circ A \dagger B \Rightarrow A \cap B=\emptyset, \\
& \circ \circ A \dagger B \quad \text { and } \quad B \subset B^{\prime} \subseteq(S \backslash A) \quad \Rightarrow \quad A \dagger B^{\prime} .
\end{aligned}
$$

A related pair $A \dagger B$ is called a Radon partition. A pair of disjoint subsets $\alpha, \beta \subseteq S$ which are not a Radon partition are said to be separated and denoted $\alpha \mid \beta$-hence the name of the structure. The separoid is acyclic if the empty set is separated from the total one; i.e., $\emptyset \mid S$.

Examples of separoids can be found in graphs [5], in oriented matroids [3, 6], and in combinatorial convexity [4], to mention just a few (see also [7]). However, the main example to keep in mind is a family of convex sets $S=\left\{K_{1}, \ldots, K_{n}\right\}$ where a Radon partition is a disjoint pair of its subsets $A, B \subseteq S$ such that the convex hull of

R. Strausz ( $\bigotimes)$

Instituto de Matemáticas, Universidad Nacional Autónoma de México, Ciudad Universitaria, 04510 Mexico D.F., Mexico

e-mail: dino@math.unam.mx 

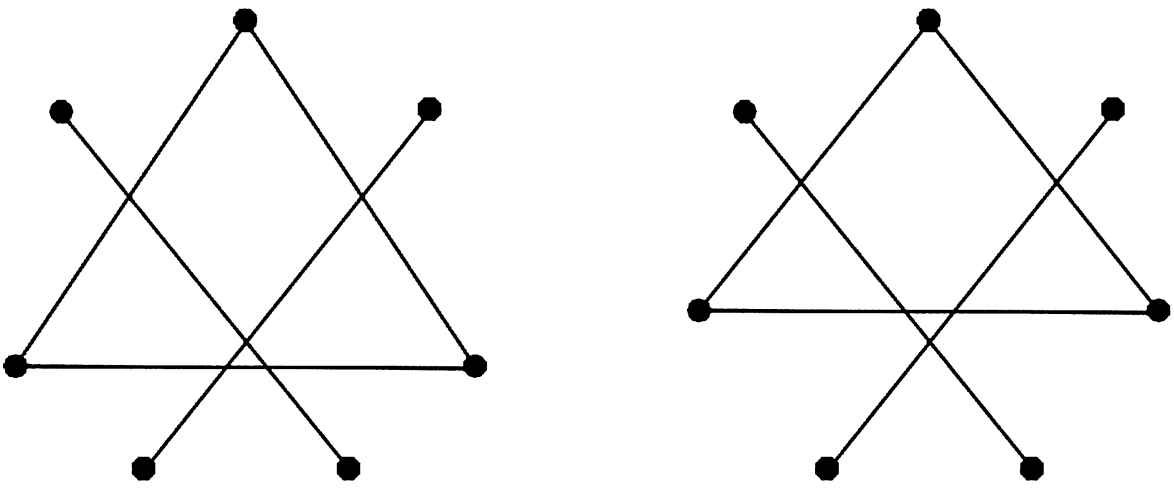

Fig. 1 Two representations of the same separoid

their unions intersect; i.e.,

$$
A \dagger B \quad \Longleftrightarrow \quad\langle\cup A\rangle \cap\langle\cup B\rangle \neq \emptyset \quad \text { (and } A \cap B=\emptyset) .
$$

Here, and in the sequel, $\langle\cdot\rangle$ denotes the convex hull operator. This is because, given a separoid $S$, there always exists a family of convex sets in the Euclidian $(|S|-1)$ dimensional space-one for each element of $S$ - such that its separoid (as defined above) is precisely $S$ (cf. [2]). This is a key ingredient in [1, 3, 6].

Observe that the separoid of a family of convex sets does not distinguish if three sets have a point in common, or if they simply intersect pairwise (see Fig. 1) - this phenomenon motivated us to introduce the notion of a virtual $\ell$-transversal in [1] and of a virtual Tverberg partition in [3].

The aim of this note is to capture such a difference in an abstract/combinatorial setting. Thus, let me introduce the following new concept (cf. [7]): a $k$-separoid is a (finite) set $S$ endowed with a symmetric $k$-relational system $\mathcal{T} \subseteq\left(\begin{array}{c}2^{S} \\ k\end{array}\right)$, defined in its family of subsets, which satisfies two simple properties: for $A_{1}, \ldots, A_{k} \subseteq S$

$$
\begin{gathered}
\circ\left\{A_{1}, \ldots, A_{k}\right\} \in \mathcal{T} \quad \Rightarrow \quad A_{i} \cap A_{j}=\emptyset \quad \text { whenever } i \neq j, \\
\circ \circ\left\{A_{1}, \ldots, A_{k}\right\} \in \mathcal{T} \quad \text { and } \quad B \subseteq\left(S \backslash \cup A_{i}\right) \quad \Rightarrow \quad\left\{A_{1}, \ldots, A_{k} \cup B\right\} \in \mathcal{T} .
\end{gathered}
$$

The elements of such a relational system will be called Tverberg partitions. Clearly, separoids are 2-separoids. The $k$-separoid is acyclic if $\left\{A_{1}, \ldots, A_{k}\right\} \in \mathcal{T} \Rightarrow$ $\prod\left|A_{i}\right|>0$.

The following discussion can be made in a more general context (for all $k>1$ ) but, to keep things simple, I will restrict to the case $k=3$-the general case is totally analogous. Thus, a Tverberg partition will be denoted simply by $A \dagger B \dagger C$. Analogously, if three pairwise disjoint subsets $\alpha, \beta, \gamma \subseteq S$ of a 3-separoid are not a Tverberg partition, they will be said to be a 3-separation and denoted by $\alpha|\beta| \gamma$.

Theorem 1 Every acyclic 3-separoid of order $n=|S|$ can be represented by a family of convex polytopes, and their Tverberg partitions, in the $(n-1)$-dimensional Euclidian space. 
Proof Let $S$ be a 3 -separoid and identify $S$ with the $n$-set $\{1, \ldots, n\}$. For each Tverberg partition $A \dagger B \dagger C$ and each element $i \in A$, assign a point of $\mathbb{R}^{n}$

$$
\mathbf{P}_{A \dagger B \dagger C}(i):=\mathbf{e}_{i}+\frac{1}{3}\left[\frac{1}{|A|} \sum_{a \in A} \mathbf{e}_{a}+\frac{1}{|B|} \sum_{b \in B} \mathbf{e}_{b}+\frac{1}{|C|} \sum_{c \in C} \mathbf{e}_{c}\right]-\frac{1}{|A|} \sum_{a \in A} \mathbf{e}_{a},
$$

where $\left\{\mathbf{e}_{i}\right\}$ denotes the canonical basis, and realize each element $i \in S$ as the convex hull of all such points:

$$
i \mapsto \mathcal{K}_{i}:=\left\langle\mathbf{P}_{A \dagger B \dagger C}(i): i \in A \text { and } A \dagger B \dagger C\right\rangle .
$$

Observe that these convex polytopes lie in the $(n-1)$-dimensional affine subspace of $\mathbb{R}^{n}$ spanned by the basis because $(*)$ is, in fact, an affine combination.

To prove that this construction is correct, two steps are needed. First, a straightforward calculation shows that the construction is made to guarantee that the Tverberg partitions are preserved; that is, consider a partition $A \dagger B \dagger C$ and observe that the vertices of the simplices $\left\langle\mathbf{e}_{a}: a \in A\right\rangle,\left\langle\mathbf{e}_{b}: b \in B\right\rangle$, and $\left\langle\mathbf{e}_{c}: c \in C\right\rangle$ "translate" to realize such a partition while intersecting precisely in their baricentre, i.e.,

$$
\begin{aligned}
\frac{1}{|A|} \sum_{a \in A} \mathbf{P}_{A \dagger B \dagger C}(a) & =\frac{1}{|B|} \sum_{b \in B} \mathbf{P}_{B \dagger C \dagger A}(b)=\frac{1}{|C|} \sum_{c \in C} \mathbf{P}_{C \dagger A \dagger B}(c) \\
& =\frac{1}{3}\left(\frac{1}{|A|} \sum_{a \in A} \mathbf{e}_{a}+\frac{1}{|B|} \sum_{b \in B} \mathbf{e}_{b}+\frac{1}{|C|} \sum_{c \in C} \mathbf{e}_{c}\right),
\end{aligned}
$$

and therefore

$$
\left\langle\bigcup_{a \in A} \mathcal{K}_{a}\right\rangle \cap\left\langle\bigcup_{b \in B} \mathcal{K}_{b}\right\rangle \cap\left\langle\bigcup_{c \in C} \mathcal{K}_{c}\right\rangle \neq \emptyset .
$$

On the other hand, to prove that the k-separations are also preserved, the following well-known fact is used: compact convex sets $K_{1}, \ldots, K_{k}$ in $\mathbb{R}^{d}$ have no point in common if and only if there are open semispaces $\ell_{+}^{1}, \ldots, \ell_{+}^{k}$ such that $K_{i} \subset \ell_{+}^{i}$ (for every $i=1, \ldots, k)$ and $\bigcap \ell_{+}^{i}=\emptyset$. The case $k=2$ is the basic separation theorem, and the general case follows by induction.

Now, consider a 3-separation $\alpha|\beta| \gamma$, and define the affine extension $\psi=$ $\psi_{\alpha|\beta| \gamma}: \mathbb{R}^{n} \rightarrow \mathbb{R}^{2}$ of the following equations: for $j=1, \ldots, n$,

$$
\psi\left(\mathbf{e}_{j}\right):= \begin{cases}\mathbf{u} & \text { if } j \in \alpha, \\ \mathbf{v} & \text { if } j \in \beta, \\ \mathbf{w} & \text { if } j \in \gamma, \\ \mathbf{0} & \text { otherwise }\end{cases}
$$

where

$$
\mathbf{u}:=\left(\begin{array}{l}
1 \\
0
\end{array}\right), \quad \mathbf{v}:=\frac{1}{2}\left(\begin{array}{c}
-1 \\
\sqrt{ } 3
\end{array}\right) \quad \text { and } \quad \mathbf{w}:=\frac{1}{2}\left(\begin{array}{c}
-1 \\
-\sqrt{3}
\end{array}\right) .
$$

Observe that $\mathbf{u}, \mathbf{v}$, and $\mathbf{w}$ are the vertices of a regular triangle with centre at $\mathbf{u}+\mathbf{v}+$ $\mathbf{w}=\mathbf{0}$. 
It follows from the definitions $(*)$ and $(* *)$-and with a little abuse of the notation-that

$$
\begin{aligned}
\psi\left(\mathbf{P}_{A \dagger B \dagger C}(i)\right)= & \psi\left(\mathbf{e}_{i}\right)-\frac{2}{3|A|}\left(\begin{array}{l}
|A \cap \alpha| \\
|A \cap \beta| \\
|A \cap \gamma|
\end{array}\right) \cdot\left(\begin{array}{c}
\mathbf{u} \\
\mathbf{v} \\
\mathbf{w}
\end{array}\right) \\
& +\frac{1}{3|B|}\left(\begin{array}{c}
|B \cap \alpha| \\
|B \cap \beta| \\
|B \cap \gamma|
\end{array}\right) \cdot\left(\begin{array}{c}
\mathbf{u} \\
\mathbf{v} \\
\mathbf{w}
\end{array}\right) \\
& +\frac{1}{3|C|}\left(\begin{array}{c}
|C \cap \alpha| \\
|C \cap \beta| \\
|C \cap \gamma|
\end{array}\right) \cdot\left(\begin{array}{c}
\mathbf{u} \\
\mathbf{v} \\
\mathbf{w}
\end{array}\right)
\end{aligned}
$$

Denote by $\psi_{\alpha}:=\psi\left(\mathbf{P}_{A \dagger B \dagger C}(i)\right)$ whenever $i \in \alpha$, and analogously with $\beta$ and $\gamma$. Then, it follows from (1) that

$$
\begin{aligned}
\psi_{\alpha} \cdot \mathbf{u}= & 1-\frac{2|A \cap \alpha|-(|A \cap \beta|+|A \cap \gamma|)}{3|A|} \\
& +\frac{|B \cap \alpha|-\frac{1}{2}(|B \cap \beta|+|B \cap \gamma|)}{3|B|} \\
& +\frac{|C \cap \alpha|-\frac{1}{2}(|C \cap \beta|+|C \cap \gamma|)}{3|C|} \\
\geq & 0,
\end{aligned}
$$

If we have that $\psi_{\alpha} \cdot \mathbf{u}>0$ and $\psi_{\beta} \cdot \mathbf{v}>0$ and $\psi_{\gamma} \cdot \mathbf{w}>0$, we are done (the semispaces $\psi^{-1}\left(\mathbf{u}_{+}^{\perp}\right), \psi^{-1}\left(\mathbf{v}_{+}^{\perp}\right)$, and $\psi^{-1}\left(\mathbf{w}_{+}^{\perp}\right)$ will do). So let us suppose, without loss of generality, that $\psi_{\alpha} \cdot \mathbf{u}=0$.

It follows from (2) that $\psi_{\alpha} \cdot \mathbf{u}=0$ if and only if $A \subseteq \alpha$ and $B \subseteq \beta \cup \gamma$ and $C \subseteq \beta \cup \gamma$. In such a case, we also have that

$$
\psi_{\beta} \cdot \mathbf{v}=1+\frac{1}{3}+\frac{1}{3}\left[\left(\frac{|B \cap \beta|}{|B|}+\frac{|C \cap \beta|}{|C|}\right)-\frac{1}{2}\left(\frac{|B \cap \gamma|}{|B|}+\frac{|C \cap \gamma|}{|C|}\right)\right] \geq 1
$$

and, analogously, $\psi_{\gamma} \cdot \mathbf{w} \geq 1$. Then we can pick any small number $0<\epsilon<1$, define the semispaces

$$
\begin{aligned}
& \ell_{+}^{\alpha}:=\left\{\mathbf{x} \in \mathbb{R}^{2}: \mathbf{x} \cdot \mathbf{u}>-\epsilon\right\}, \\
& \ell_{+}^{\beta}:=\left\{\mathbf{x} \in \mathbb{R}^{2}: \mathbf{x} \cdot \mathbf{v}>1-\epsilon\right\}, \\
& \ell_{+}^{\gamma}:=\left\{\mathbf{x} \in \mathbb{R}^{2}: \mathbf{x} \cdot \mathbf{w}>1-\epsilon\right\},
\end{aligned}
$$

and their preimages $\psi^{-1}\left(\ell_{+}^{\alpha}\right), \psi^{-1}\left(\ell_{+}^{\beta}\right)$, and $\psi^{-1}\left(\ell_{+}^{\gamma}\right)$ will do the work, concluding the proof.

Observe that, as the Radon partitions do not contain information on the Tverberg partitions (cf. [3]), the 3-separoid also loses some information on the 2-separoid; e.g., 
consider 5 (different) points in the line and give them the order $(1,2,3,4,5)$. These points have the following two Tverberg partitions:

$$
15 \dagger 24 \nmid 3 \text { and } 14 \dagger 25 \dagger 3 \text {. }
$$

After the representation of Theorem 1, the configuration $\left\{\mathcal{K}_{i}: i=1, \ldots, 5\right\}$ will lose, among others, the Radon partition $2 \nmid 13$. To correct this "anomaly" we can go one step further and define a hyperseparoid as a set $S$ endowed with a collection of families of subsets $\mathcal{T} \subseteq 2^{2^{S}}$ which satisfies, for $A_{1}, \ldots, A_{k} \subseteq S$,

$$
\begin{aligned}
& \circ\left\{A_{1}, \ldots, A_{k}\right\} \in \mathcal{T} \quad \Rightarrow \quad\left\{A_{1}, \ldots, A_{k-1}\right\} \in \mathcal{T}, \\
& \circ \circ\left\{A_{1}, \ldots, A_{k}\right\} \in \mathcal{T} \quad \Rightarrow \quad A_{i} \cap A_{j}=\emptyset \quad \text { whenever } i \neq j, \\
& \circ \circ \circ\left\{A_{1}, \ldots, A_{k}\right\} \in \mathcal{T} \quad \text { and } \quad B \subseteq\left(S \backslash \cup A_{i}\right) \quad \Rightarrow \quad\left\{A_{1}, \ldots, A_{k} \cup B\right\} \in \mathcal{T} .
\end{aligned}
$$

Clearly, a similar construction to that of Theorem 1 will lead to a representation of hyperseparoids by convex polytopes and their Tverberg (and Radon) partitions.

Let us close this note with a couple of challenging open questions. In the spirit of [2], when can a hyperseparoid be represented by points (instead of convex sets)? Given a hyperseparoid, can we decide in polynomial time if it can be represented by points?

\section{References}

1. Arocha, J.L., Bracho, J., Montejano, L., Oliveros, D., Strausz, R.: Separoids, their categories and a Hadwiger-type theorem for transversals. Discrete Comput. Geom. 27, 377-385 (2002)

2. Bracho, J., Strausz, R.: Two representation theorems for separoids. Period. Math. Hung. 53, 115-120 (2006)

3. Montellano-Ballesteros, J.J., Pór, A., Strausz, R.: Tverberg-type theorems for separoids. Discrete Comput. Geom. 35, 513-523 (2006)

4. Montellano-Ballesteros, J.J., Strausz, R.: Counting polytopes via the Radon complex. J. Comb. Theory, Ser. A 106, 109-121 (2004)

5. Nešetřil, J., Strausz, R.: Universality of separoids. Arch. Math. (Brno) 42, 85-101 (2006)

6. Strausz, R.: Erdős-Szekeres "Happy-end"-type theorems for separoïds. Eur. J. Comb. 29(4), 1076-1085 (2008)

7. Strausz, R.: On separoids. Ph.D. Thesis, Universidad Nacional Autónoma de México (2004). Also available at http://www.math.unam.mx/strausz/Papers/DrThesis.pdf 